\title{
From the Editor's Desk: Our Paper Anniversary
}

\author{
Mitchell D. Feldman, MD, MPhill and Richard L. Kravitz, MD, MSPH ${ }^{2}$ \\ 'Division of General Internal Medicine, University of California, San Francisco, San Francisco, CA, USA; ${ }^{2}$ UC Davis Division of General Medicine, \\ University of California at Davis, Sacramento, CA, USA.
}

\author{
$J$ Gen Intern Med 25(12):1259-65 \\ DOI: $10.1007 / \mathrm{s} 11606-010-1528-1$ \\ (C) The Author(s) 2010. This article is published with open access at \\ Springerlink.com
}

$\mathrm{T}$ radition has it that first anniversaries are celebrated with gifts of paper. Thus, it is fitting that on this first anniversary of our start as co-Editors in Chief of the Journal of General Internal Medicine, we should look back on the 12 paper issues we've published in 2010. The paper journal is sent to every member of the Society of General Internal Medicine and to a diminishing number of libraries who still choose to display the bound journal instead of relying solely on electronic access. As editors, we take the print journal seriously, striving to craft each issue to be a self-contained and coherent whole.

This year has seen the addition of several new features that we hope add value to each issue. From the Editor's Desk directs readers' attention to articles and themes of special interest. Exercises in Clinical Reasoning examines the diagnostic process as highly skilled clinicians work through a tough clinical case. Innovations and Improvement explores the process of quality improvement and enlivens the journal through interviews and first person narratives, and Healing Arts sounds the lived experience of general internal medicine in essays, poems, and criticism.

Although we choose the articles appearing in each issue of the print journal with care, most consumers of medical journals never touch the paper of each monthly issue and instead prefer to access JGIM and other journal content as if it were on iTunes, searching through titles and abstracts and downloading individual articles. They find JGIM content through on-line sources such as Google Scholar, PubMed Central, and others. In fact, over the past year, while Springer, our publisher, mailed just over 2,000 copies of the paper journal to subscribers or members each month, there have been more than 1 million JGIM articles downloaded by readers

Published online October 7, 2010 since the publication of our inaugural issue last January. This, of course, is the present and future of medical journals; the increasing 'webification' of journal content and the growing irrelevance of the print journal. We are well aware of these trends and will work over the remaining 4 years of our editorship to prepare JGIM for the inevitable demise of the print journal and the predominance of the web-based journal.

With the help of Associate Editor Malathi Srinivasan and others, work on eJGIM has already begun. In addition, the next 12 months will see the reinvigoration of Innovations in Medical Education, a stunning series of articles on Health Policy, and new collaborations with AHRQ, the VA, and the Annals of Internal Medicine.

JGIM depends on the generosity of a committed and outstanding group of reviewers. In 2009-2010, JGIM reviewers volunteered their time and expertise to review about half of the more than 1,000 manuscripts that JGIM receives each year. During this period, 986 reviewers provided a total of 1,294 reviews with a mean quality score of 2.98 on a scale of 1-6 (as judged by JGIM Deputy Editors). Of these, 276 provided at least two reviews, and 29 provided three or more. We are indebted to them for their service.

Among this group of dedicated peer reviewers, there is a cohort that stands out. Reviewers included in this prestigious group performed at least two reviews between July 2009 and June 2010, returned all reviews within 30 days, and received no quality score on any review lower than 4 on our 1-6 scale. An asterisk identifies the 131 reviewers meeting these criteria. We thank them for their efforts on behalf of the Journal.

In our first "From the Editor's Desk" in January 2010 we wrote: "In steering the journal forward over the next 5 years, we will be guided by the principle that JGIM is a journal for generalists committed to improving the world in which they practice and teach. Thus, we will seek to publish data derived from settings where real patients live and real doctors practice, as well as reviews and tools that clinicians and educators can use to do their jobs more effectively, efficiently, and humanely." We remain committed to this vision over the next 4 years and hope that you, our readers, will join us by continuing to submit to, review for, read, and respond to JGIM. Whether in print or on-line, JGIM is your journal, and we need to hear from you. Send us an e-mail (or a paper letter!) and share your thoughts for the future of medical journalism and the future of medicine.

The Editors 


\section{JGIM REVIEWERS, 2009-2010}

Aagaard, Eva*

Abbo, Elmer

Abou-Raya, Anna

Acheson, Louise

Ackerman, Alexandra

Acosta, David*

Aday, Ron

Ahluwalia, Jasjit

Akrawinthawong, Krittapoom

Albert, Nancy

Alexander, Stewart

Allen, Adrienne*

Alley, Dawn

Alvanzo, Anika

Ancelin, Marie L.

Anda, Robert

Andermann, Anne

Anderson, Daren*

Anderson, Karen

Anderson, Lynda

Anderson, Robert

Anderson, Wendy

Andresen, Elena

Ang, Dennis

Ansari, Muhammad

Ansell, Jack

Antommaria, Armand

Apalara, Jelili

Aparasu, Rajender

Applegate, Kimberly

Arayssi, Thurayya

Armenian, Saro

Armstrong, Mary Anne

Arneson, Tom*

Aronow, Wilbert

Aronson, Louise

Arora, Vineet*

Ash, Arlene

Assmann, Susan

Attridge, Russell

Aulakh, Sudeep*

Ayas, Najib

Babalola, Adeniyi

Baernstein, Amy

Bagchi, Ann

Baine, William*

Bair, Matthew

Baker-Genaw, Kimberly

Ball, Daniel

Banday, Wamiq

Banta, Jim*

Barg, Frances*

Barnard, David

Barr, Michael*

Barrett, Michael

Barron, Beth

Barsuk, Jeffrey

Basner, Mathias

Bassett, Ingrid

Batch, Bryan
Bates, Carol

Bates, David

Batsis, John

Bauer, Doug

Beach, Mary Catherine*

Becker, Bryan*

Beckman, Thomas

Bell, Christina*

Bender, D.

Berger, Barbara

Berkenblit, Gail

Berkman, Nancy

Bern-Klug, Mercedes*

Bernstein, Lisa

Berry, Stephen

Best, Jennifer

Betancourt, Joseph

Bhimaraj, Arvind*

Bierer, Beth

Bissell, Michael

Blanco, Carlos

Blue, Amy*

Blustein, Jan

Bodenheimer, Thomas

Boguradzka, Anna

Bokhour, Barbara

Bolen, Shari

Boltri, John

Bonner, Michael

Boockvar, Kenneth

Boonyasai, Romsai

Borowsky, Steve

Borrell, Luisa

Boser, Susan

Boulware, L. Ebony

Boyd, Cynthia

Braddock, Clarence

Bradley, Elizabeth

Branch, William

Branson, Bernard*

Breite, Ira

Brewster, Joan

Bridges, John

Brook, Robert

Brophy, Jay

Buchanan, David

Buczko, William*

Bungay, Kathleen

Burge, Russel

Burgess, Diana

Burton, Michael

Butler, J.S.

Butler, James

Bynum, Julie

Caccamese, Suzanne

Cadena, Jose

Calhoun, Elizabeth

Cameron, Kenzie

Campbell, Noll

Cappelleri, Joseph

Carek, Peter

Carlson, Kathleen
Carney, Stacie

Carrico, Adam

Cavanaugh, Kerri

Cegala, Donald

Cene, Crystal

Champion, Howard

Chan, An-Wen

Chao, Samantha

Chapman, Gretchen

Charles, Cathy

Charlson, Mary

Chaudhry, Amina

Cheng, Eric

Cheng, Hugo

Chenot, Jean-Francois

Cherniack, (Evan) Paul*

Chernof, Bruce Allen*

Cherrington, Andrea

Cheskin, Lawrence

Chin, Jeanette

Chin, Marshall

Chopra, Deepti

Chou, Calvin

Chou, Roger*

Choudhry, Niteesh

Chretien, Katherine*

Chu, Pei-Yi

Chudgar, Saumil

Clark, Alexander

Clark, Cheryl

Clay, Michael*

Clayton, Charles*

Cohen, Alan

Cohen, Ellen

Cohen, Joshua

Cohen, Pieter

Coleman, Kimberly

Coleman, Margaret

Collins, Mary Elizabeth

Compton, Michael

Concannon, Thomas

Connelly, Julia

Cooper, Lisa

Corbie-Smith, Giselle

Cordasco, Kristina

Coups, Elliot

Cram, Peter

Crandall, Lee

Crandall, Sonia

Criley, Jasminka

Cronholm, Peter

Cruess, Richard

Cuerdon, Timothy

Cully, Jeffrey

Culpepper, Larry

Cunningham, Kelly

Cunningham, Peter

Cutrona, Sarah

D'eramo-Melkus, Gail

David, Alan

David, Michele

Davis, Esa* 
Davis-Smith, Monique

Dawood, Nazeera

De Lissovoy, Gregory

De Vries, Esther*

Dedios, Maria Cecilia

Denshaw-Burke, Mary

Desai, Rani

Desroches, Catherine

Devkota, Bishnu

Dexter, Paul

Dhurandhar, Anjali

Diamond, James

Diamond, Lisa

Didwania, Aarati

Diehl, Andrew

Dietzen, Diane

Dimatteo, Livio

Dimitropoulos, Linda

Dine, Jessica*

Djulbegovic, Benjamin

Djuricich, Alexander

Dobie, Sharon

Dobscha, Steven

Dohan, Daniel

Dolan, James

Dolovich, Lisa

Donato, Anthony*

Dong, Yue

Donohue, Julie

Dorr, David

Dresselhaus, Timothy

Dublin, Sascha*

Dudzinski, Denise

Dugan, Elizabeth

Dunton, Genevieve

Duru, O. Kenrik

Edwards, W. Sherman*

Eisen, Susan

Ell, Kathleen

Ellis, Charles*

Elstein, Arthur

Elwyn, Glyn

Enderle, Mark

Engelman, Kimberly

Escalante, Carmen

Escobar, Javier I.

Essink-Bot, Marie-Louise

Etchells, Edward

Fancher, Tonya

Fansler, Derrick

Farber, Jeffrey*

Farber, Neil

Fargason, Jr., Crayton

Farkas, Kathleen

Farnham, Paul G.

Federman, Douglas

Feero, W. Gregory

Fein, Oliver

Feldman, Henry

Felix, Kaytura

Felker, Bradford

Feng, Yongqing
Fennie, Kristopher

Ferguson, Kristi*

Fiebach, Nicholas

Filice, Gregory

Finkelstein, Joseph

Finney, John*

Fiscella, Kevin

Fish, Jason*

Fish, Laura

Fisher, Dennis

Fitzgerald, James

Fitzgibbons, John*

Fleisher, Lee

Fletcher, Kathlyn

Folland, Sherman

Fong, Tamara

Ford, Earl

Forjuoh, Samuel

Fox, Chris

Fox, Patrick

Fox, Rena

Fraer, Mony

Frakt, Austin

Francis, Maureen

Frazier, Susan*

Frei, Christopher

Friedman, Allon

Friedmann, Peter

Frohna, John

Fuller, Michael

Fullerton, Stephanie Malia

Funkhouser, Ellen

Gagliardi, Anna

Gagnon, Marie-Pierre

Galbraith, Alison*

Galesic, Mirta

Galindo, Michael

Gallagher, Teresa

Gandhi, Devang

Gandhi, Tejal

Ganiats, Ted

Garcia-Retamero, Rocio

Garden, Rebecca

Gardenier, Donald

Gellad, Walid

Gellad, Ziad

George, Marina

Georges, Catherine Alicia

Gierisch, Jennifer*

Gilbert, Gregory*

Gill, James

Gill, Raminder

Gilman, Boyd

Gimm, Gilbert

Ginsburg, Shiphra*

Girard, Donald

Girotti, Jorge

Glenn, Beth

Glisczinski, Marcy

Glisson, James*

Gluud, Lise Lotte

Goldsmith, Charles
Gomez, Arthur

Goodlin, Sarah

Goodson, John

Gornick, Marian

Gowd, B.M.Pampana

Graham, Jeremy

Graham, Mark

Grande, David

Granger, Carl

Granowitz, Eric V.

Grau, Lauretta

Gray, Bradley

Gray, Darryl

Green, Alexander

Green, Larry

Greenberg, Dan

Grennan, Tim

Grey, Michael

Greysen, Ryan

Griffin, Joan

Grossman, Joy

Grossman, Susan

Gruber-Baldini, Ann

Gum, Amber

Gupta, Neil

Gupta, Ridhi

Gupta, Sameer

Gupta, Samir*

Haggstrom, David

Hahn, Elizabeth

Haidet, Paul

Hall, Karen

Hallenbeck, James

Hamieh, Tarek

Hamm, Robert

Hamman, Richard

Hanchate, Amresh*

Hanes, Pamela

Hanoch, Yaniv

Hanson, Brenda

Hanson, Janice

Harada, Nancy

Harris, Julie

Harris, Patricia

Harrison, Margaret

Harrison, Van

Hartmann, Christine*

Hastings, James*

Hatahet, Mohamad

Hatem, David

Havyer, Rachel*

Hawley, Sarah

Hayes, Patricia

Hays, J. Taylor

Heckbert, Susan

Heisler, Michele

Helft, Paul

Helmer, Drew

Hensley Alford, Sharon

Herbeck Belnap, Bea*

Heritage, John

Heslin, Kevin 
Hess, Lisa

Hewett, David

Hinchey, Kevin

Hinchey, Sherri*

Hirsch, Calvin

Hirsh, Joel

Hirshfield, Sabina

Hoenig, Helen

Hoffman, Kimberly*

Hogan, Timothy

Holden, Debra

Holl, Jane

Holman, Halsted*

Holmes, Ann M.

Holmes, Holly

Holtman, Matthew*

Holzer, Gerold

Hooker, Roderick

Horner, Ronnie

Horsley, Tanya

Horvitz-Lennon, Marcela

Howell, Elizabeth

Huang, Elbert*

Huddle, Thomas

Hudson, Shawna

Huggett, Kathryn

Huizinga, Mary*

Huot, Stephen

Hynes, Denise

Iftikhar, Salma

Ijaz, Nadia

Inagami, Sanae

Insinga, Ralph

Inui, Thomas

Iwashyna, Theodore

Jack, Brian

Jackson, George

Jacobs, Rowena

Jacobson, Kara

Jaeger, Jeffrey

Jain, Sharad

Jaitly, Manasvi

Jalbert, Jessica

James, Aimee*

Jin, Justin

Jo, Angela

Johl, Karnjit

Johnson, Daniel

Johnson, Julie*

Johnson, William

Johnston, Katherine

Jolly, Brian

Jolly, Stacey

Jones, Mark

Joseph, Djenaba

Justiss, Michael D.

Justo, Dan

Juul, Dorthea

Kado, Deborah

Kahan, Scott

Kahlon, Gunjan

Kahn, Marc
Kaissi, Amer

Kandpal, Saurabh

Kandula, Namratha

Kanjwal, Mohammad

Kansagara, Devan*

Kaphingst, Kimberly

Kapoor, Alok

Kapp, Julie*

Karan, Mehmet Akif

Karon, Sarita

Kartha, Anand

Kassirer, Jerome

Kavalieratos, Dio

Kegler, Michelle

Kehle, Shannon*

Keranen, Lisa

Kermani, Tanaz

Kertesz, Stefan

Kerwin, Diana

Kesselheim, Aaron

Kessler, Ronald

Kho, Abel

Kikano, George

Killeen, Ronan

Kilo, Charles

Kim, Karen

Kim, Sara

Kim, Sarang

Kim, Scott

Kim, Theresa

Kimberly, John

Kinghorn, Warren

Kinney, Eleanor

Kirchner, Joann

Kirk, Julie

Kittisupamongkol, Weekitt

Kizer, John

Klein, Robin*

Kleshinski, James

Knaus, William

Knight, Chris

Koetting, Michael

Kogan, Jennifer

Koppel, Ross

Koracevic, Goran

Korenstein, Deborah

Kramer, M. Kaye

Kraschnewski, Jennifer*

Krebs, Erin

Krebs-Smith, Susan

Kremer, Heidemarie

Kress, John

Kripalani, Sunil

Kripke, Daniel

Kronish, Ian

Kukla, Rebecca

Kullgren, Jeff*

Kumagai, Arno

Kumar, Gautam

Kutner, Nancy

Kuver, Rahul

Labarère, José
Lai, Cindy

Lakey, Susan

Lamantia, Michael

Landman, Adam

Landon, Bruce*

Lantos, John

Lanzkron, Sophie

Larson, Eric

Lauderdale, Diane

Lawson, Kenneth

Lazar, Lawrence*

Lazarus, Cathy

Lee, Gunjeong*

Lee, Joshua

Lee, Margaret

Lee, Pearl*

Lee, Rita*

Lee, Thomas

Lemak, Christy Harris

Lemke, Sonne*

Lemon, Maurice

Leng, Sean

Lenth, Russell

Lepore, Michael

Lerner, Jeffrey

Leventhal, Howard

Levy, Cari

Levy, Lawrence

Lewis, Carmen

Lewis, Keir

Leykum, Luci

$\mathrm{Li}$, Alexander

Li, Pengxiang

Liang, Wenchi

Lichstein, Peter

Lie, Desiree*

Lin, Grace

Lin, James

Lin, Jenny

Lin, Ming Valerie

Linas, Benjamin

Liu, Chuan-Fen

Llorca, Javier

Lo, Margaret

Locke, Charles

Loeb, Susan

Logio, Lia

Long, Judith

Lorig, Kate

Lotufo, Paulo

Lu, Lee

Luke, Roice*

Lukela, Michael

Lyles, Alan

Lynch, Cheryl

Machtinger, Edward

Maciejewski, Matthew

Maciosek, Michael

Magura, Stephen

Magwood, Gayenell

Maher, Chris

Mai, Cuc 
Manchikanti, Laxmaiah

Manfred, Lynn

Manganello, Jennifer

Manjarrez, Efren

Mann, Devin

Manning, Dennis

Mao, Jun

Markoff, Brian

Marks, Guy

Martin, Erika*

Marusic, Ana

Maruthur, Nisa

Masi, Christopher

Masica, Andrew

Matthias, Marianne

Maurer, Martha

Mavis, Brian

Mccall-Hosenfeld, Jennifer

Mccaw, Brigid

Mccullough, Peter

Mccusker, Jane

Mcdonald, Charlotte

Mcdonald, Mba, Kathryn

Mcdougle, Leon

Mcglynn, Elizabeth*

Mcguire, Amy

Mckellar, John

Mcknight, Patrick

Mcneely, Jennifer

Mcwilliams, J. Michael

Mechaber, Hilit

Meckley, Lisa

Meerschaert, M. Carmen

Mehta, Manish

Meka, Praveen

Mello, Michelle

Merrill, Angela

Metzger, Maureen

Meyers, David

Michaelson, James

Michota, Frank

Miller, Doriane

Min, Lillian

Misra, Ranjita

Mokwunye, Nneka*

Mondragon, Delfi

Montello, Martha

Moody-Ayers, Sandra

Mookherjee, Somnath*

Moon-Howard, Joyce

Mopala, Prashanth

Moran, William

Morasco, Benjamin

Moreno, Lorenzo*

Moskowitz, Alan

Mourad, Michelle*

Mueller, Martina

Murarka, Shishir

Murff, Harvey

Musick, David

Nadkarni, Anagha

Naik, Chetan*
Narang, Jatinder*

Nease, Donald

Neely, David

Nekhlyudov, Larissa

Nelson, David

Nelson, Karin

Neuhauser, Duncan

Neuner, Joan

Newcomer, Robert

Newton, Katherine

Nguyen, Huong

Nguyen, Long

Nichols, Linda

Niederdeppe, Jeff

Nieman, Linda

Niles, Barbara*

Njike, Valentine

Nunez, Ana*

Nunez-Smith, Marcella

Nutting, Paul

Nyweide, David

O'brien, Bridget*

O'leary, Kevin*

O'malley, Ann*

O'malley, Cheryl

O'sullivan, Patricia*

Ogrinc, Greg*

Okumura, Megumi

Opole, Isaac

Orlando, Lori

Osborn, Chandra*

Osilla, Karen

Oudega, Ruud

Overholser, Linda

Owen-Smith, Amanda

Ozanne, Elissa

Pagoto, Sherry*

Palmas, Walter

Palmer, Richard

Pandey, Rahul

Pandey, Tanu

Pangaro, Louis

Paniagua, Angela

Papageorgiou, Panos

Parikh, Nina

Parikh, Puja

Parikh, Sameer

Pasick, Rena

Patel, Mital*

Patel, Mitesh

Pathman, Donald

Pawlson, Greg

Peccoralo, Lauren

Peek, Monica

Peikes, Deborah

Pena, Adolfo

Perkins, Henry

Perkowski, Linda

Pernod, Gilles

Persad, Govind

Persell, Stephen

Pescosolido, Bernice
Petersen, Amy

Peterson, Neeraja

Petittii, Diana

Pham, Phuong-Chit

Pickering, Joyce

Pierce, John

Pirraglia, Paul

Pliskin, Joseph

Poleshuck, Ellen

Politi, Mary

Pollack, Craig*

Post, Edward

Powell, Adam*

Powell, Heidi

Prentice, Julia

Prochaska, Judith

Rabi, Doreen

Radosevich, David

Rahman, Abu

Rajan, Sabitha

Ramachandra Pai, Rajasree Pai

Ramakrishnan, Kalyanakrishnan

Ramaswamy, Anuradha

Ramirez, Mildred

Ranji, Sumant

Rascati, Karen

Ratuapli, Shiva

Raue, Patrick

Razafsha, Mahdi

Rector, Thomas

Reddy, Shalini*

Redmond, Nicole*

Reed, Mary

Reerink, Evert

Rehman, Shakaib

Reimer, Christie*

Reis, Shmuel

Rejnmark, Lars

Rennhoff, Christina

Rhoades, Jeffrey

Rich, Eugene*

Riddle, Janet

Riesen, Walter

Riessman, Catherine

Ristvedt, Stephen*

Rivera, Cristina*

Robinson, Jeffrey

Roddy, Juliette

Rodriguez, Carlos

Rohrer, James

Rootman, Irving*

Rose, Adam*

Rosenbaum, Julie

Rosenblum, Michael

Ross, Joseph

Rothman, Russell

Rouan, Gregory*

Rouf, Emran

Roy, Christopher

Roy, Michael

Ruser, Christopher

Rushakoff, Robert 
Russell, Daniel

Rutter, Carolyn

Sacco, William

Sahai, Sunil

Saigh, Orit

Saini, Sameer

Saliba, Debra

Saluja, Sandeep

Samet, Jeffrey

Sandars, John

Sanderson, Saskia

Sarkar, Urmimala

Scalese, Ross

Scanlon, Dennis

Schaffer, Adam*

Schardt, Connie

Schatz, Michael

Scheffler, Richard*

Schenker, Yael*

Scherer, Martin

Schilling, Lisa*

Schmid, Arlene

Schmidt, John

Schneider, John

Schoen, Cathy

Schoenbaum, Stephen*

Schoenthaler, Antoinette*

Schonberg, Mara*

Schulberg, Herbert

Schulman-Green, Dena

Schultz, Henry

Schumacher, Gerald

Schumann, John

Schuwirth, Lambert

Schwartz, Alan*

Schwenk, Thomas

Scott, Eric*

Seago, Jean Ann

Sefcik, Susan

Segura, Jennifer

Sehgal, Ash

Semaan, Salaam

Sessums, Laura

Sethi, Arjinder

Shah, Chirayu*

Shah, Nirav R.

Shapiro, Johanna

Sharma, Priyanka

Shavers, Vicki

Shea, Dennis

Shea, Judy

Shimada, Stephanie

Ship, Amy

Shokar, Navkiran

Shoptaw, Steven

Shunk, Rebecca

Sifri, Randa

Simmons, Ashley

Simon, Daniela

Simon, Melissa

Sin, Don

Sinaiko, Anna
Singer, Eleanor

Singer, Ken

Singh, Hardeep

Singh, Jasvinder

Singh, Simran

Sirio, Carl

Skaer, Tracy

Sliem, Hamdy

Smith, Alexander

Smith, Dustin

Smith, Lawrence

Smith, Patricia*

Smith, Peter

Smith, Robert

Snowden, Lonnie

Snyder, Claire

Solberg, Leif

Solomon, Jeffrey*

Solomon, Matthew

Sosman, James

Soule, Jeremy

Spangler, Leslie*

Spoont, Michele

Sprague, Carol

Stamps, Paula

Stapleton, Ann

Starfield, Barbara

Starrels, Joanna*

Stefan, Mihaela

Steinman, Michael*

Stevens, David*

Stevens, Scott*

Stoddard, Anne

Stoddard, Hugh

Stone, Valerie

Strassels, Scott

Strauss, Jennifer

Strauss, Ronald

Street, Richard*

Stuart, Brad

Studdert, David

Subramanian, Sujha

Sudore, Rebecca

Sumner, Walton

Susman, Jeff

Sussman, Andrew*

Symm, Barbalee

Szaflarski, Magdalena

Szostek, Jason

Tai-Seale, Ming

Tamblyn, Robyn

Taminiau-Bloem, Elsbeth

Tang, Joyce

Tangri, Navdeep

Tarn, Derjung*

Taveira, Tracey*

Taylor, Brent*

Teherani, Arianne

Teitelbaum, Joel

Telfair, Joseph

Terman, Eric

Tetrault, Jeanette
Thaler, David

Thielke, Stephen*

Thirumaran, Rajesh

Thompson, George

Thornton, John*

Tilburt, Jon

Timbie, Justin

Tjia, Jennifer

Tleyjeh, Imad

Torke, Alexia

Trauth, Jeanette

Trepka, Mary Jo

Trikalinos, Thomas

Triola, Marc

Tsai, Adam

Tsai, Alexander

Tsao, Yu-Tzu

Tune, Larry

Tyssen, Reidar

Uchida, Toshiko

Udow, Marianne

Uhrig, Jennifer

Urban, Susan

Vacek, James

Vachharajani, Tushar

Valentine, Christopher

Valenzuela, Terence

Van Walraven, Carl

Vassy, Jason*

Vaughn, Thomas

Vawter, Dorothy

Veeranna, Vikas*

Vega, William

Vela, Monica

Venkitachalam, Lakshmi

Vijan, Sandeep

Vinson, Daniel

Viswanathan, Meera

Vohra, Sunita

Voils, Corrine

Volpato, Stefano

Wagner, Ed

Wagner, Krystn

Waite, Eva

Wajnberg, Ania*

Wakefield, Bonnie

Walling, Anne

Walsh, Kathleen

Walshe, Catherine

Wang, Catharine

Wang, Virginia

Warm, Eric

Washington, Donna

Wathen, Patricia

Wear, Delese

Weckmann, Michelle

Wehby, George

Weiser, Sheri

Weiss, Linda

Weissman, Joel

Weller, Wendy

Wenzel, Suzanne 
Werner, Rachel

Wess, Mark

West, Bernadette

West, Eileen

Wetmore, James

Wetterneck, Tosha

White, Becky

White, Douglas

White, Katie

White, Mary

Whiteman, Douglas

Whitley, Elizabeth

Whitney, Simon

Whitson, Heather*

Widera, Eric*

Widome, Rachel

Wiese, Jeffrey

Wilkerson, Luann

Willett, Laura

Williams, Karen Patricia

Williams, Linda

Wilson, Stephen
Wilson-Stronks, Amy

Windish, Donna

Wittich, Christopher

Wong, Eunice

Wood, Malissa

Wood, Timothy*

Woodard, Lechauncy

Wooldridge, David

Woolhandler, Steffie

Wright, Scott

Wynia, Matthew

Yadav, Surinder

Yancey, Antronette

Yang, Frances

Yanni, Leanne

Yarborough, William

Ye, Jiali

Ye, Lichuan

Yedla, Parekha

Yegneswaran, Balaji

Yelin, Ed

Yi, Jenny
Yi, Michael

Yood, Robert

Young, Henry

Young, Meredith

Zaas, Aimee

Zafar, Atif

Zema, Carla

Zuberi, Rukhsana

Zulman, Donna*

*Superior Reviewer, defined as $>=2$ reviews, quality score no lower than 4 of 6 on any single review, no review returned past 30 days

Open Access: This article is distributed under the terms of the Creative Commons Attribution Noncommercial License which permits any noncommercial use, distribution, and reproduction in any medium, provided the original author(s) and source are credited. 